Herausgeber:

Zentralinstitut für Ernăhrung der Akademie der Wissenschaften der DDN Direktor: Prof. Dr. habil. II. Haene

in Zusammenarbeit mit

der Gesellschaft für Ernāhrung in der DDR (Vorsitzender: Prof.Dr. habil. H.Schmandke), der Arbeitsgruppe Ernăhrung beim Nationalen Komitee für Gesundheitserziehung der DDI (Leiter: Prof. Dr. habil. H.-A. Ketz) und dem Warenzeichenverband Diätetische Erzeug nisse der DDR $\odot . V$. (Generaldirektor: F. Schmidt).

Redaktion :

Dr. Fricdbert Baum (Chefredakteur), Dr. Jürgen Proll,

Dipl.-Journ. Richard Baier.

Redaktionsbeirat :

Dr. M. Anders, Dr. sc. M. Möhr,

Dr. R. Schmelter, Dr. sc. H. E. Schmidt,

Prof. Dr. habil. M. Uhlmann, Dr. J. Voigt.

Anschrift der Redaktion :

Zentralinstitut für Ernährung der

Akademie der Wissenschaften der DDR,

DDR-1505 Bergholz-Rehbrücke,

Arthur Scheunert Allee 114-116.

Verlag:

Akademie-Verlag, DDR-108 Berlin Leipziger Str. 3-4; Fernruf 2200441 ;

Telex-Nr. 114 420; Postscheckkonto:

Berlin: 35021 ; Bank: Staatsbank der DDR. Berlin, Kto.-Nr.: 6836-26-20712,

Veröffentlicht unter dor Lizenznummer 1656 des Presseamtes beim Vorsitzenden des Ministerrates der Deutschen Demokratischen Republik.

Gesamtherstellung :

VEB Druckerei ,Thomas Müntzer “,

DDR-582 Bad Langensalza.

Erscheinungsweise :

Die Zeitschrift „Ernāhrungsforschung" erscheint jăhrlich in einem Band mit 6 Heften. Bezugspreis je Band 48,- M zuzüglich Ver sandspesen (Preis für die DDR 30, - M) Preis je Heft 8, - M (Preis für die DDR 5, - MI) Bestellnummer dieses Heftes: 1091/21/5.

Alleinige Anzeigenannahme: DEWAG-WERBUNG, DDR-1054 Berlin, Wilhelm-PiockStr. 49 und alle DEWAG-Betriebe in den Bezirksstädten der DDR.

Urheberrecht:

Den Tagegzeitungen der Deutschon Demokratischen Republik ist der auszugsweise Nachdruck der Beiträge dieser Zeitschrift bei Quellenangabe honorarfrei gestattet. Ansonsten alle Rechte vorbehalten, insbesondere die der Ubersetzung. Kein Teil dieser Zeitschrift darf in irgendeiner Form - durch Photokopic, Mrikrofilm oder irgend ein anderes Verfabren - ohne schriftliche Genehmi. gung des Verlages reproduziert werden. (C) 1976 by Akademie-Verlag Berlin. Printed in the German Democratic Republic.
Aufgaben der Ernährungswissenschaft im Rahmen der Gesundheits politik der DDR nach dem IX. Parteitag der SED . . . . . . . . . 129

Ernährung und Leistung: III. Der Einfluß der Ernährung auf die Leistungsfähigkeit im tropischen und Höhenklima sowie bei Sehwerelosigkeit . . . . . . . . . . . . . . . . . . . . . . . . . . . 133

Zur Epidemiologie des Diabetes . . . . . . . . . . . . . . . . . 136

Zinkmangel bei Frauen festgestellt . . . . . . . . . . . . . . . 138

Schüler- und Kinderspeisung in der DDR - 1971 bis 1976 . . . . . 139

Vergleichende Betrachtung der hygienisehen und ernährungshygienisehen Situation der Schülerspeisung eines Stadt- und Landkreises . . 141

Fisch in Pulverform . . . . . . . . . . . . . . . . . . . . . 145

Na, dann - Prost . . . . . . . . . . . . . . . . . . . . . . 145

Postgraduales Studium ,Lebensmittelhandel“ an der Hundelshochschule Leipzig . . . . . . . . . . . . . . . . . . . . . . . . 146

Buchbesprechung: Betricbliche Gemeinschaftsverpflegung . . . . . 147

Erfordernisse zur effektiven Gestaltung der sozialistischen Warenzirkulation bei Lebensmitteln . . . . . . . . . . . . . . . . . . 148

Tagungsbericht: Umweltverunreinigung und Krebsrisiko . . 150

Tendenzen der Adipositas-Entwieklung in der čSSR . . . . . . . . 151

Salima 76: III. Internationaler Nahrungsmittelsalon in Brno/ČSSR 152 Tagungsbericht: XIX. Kongre $\beta$ der Slowakischen Gesellschaft für rationelle Ernährung . . . . . . . . . . . . . . . . . . . . . 153

Zur Nachahmung empfohlen . . . . . . . . . . . . . . . . 154

Lebensmittel-Lexikon . . . . . . . . . . . . . . . . . . . . 155

Hilft Krill die Eiweißlücke sehließen? . . . . . . . . . . . . . . 157

Wo Freimileh zur Problemmileh wird . . . . . . . . . . . . . . 157

Buchbesprechung: Internationale Probleme der modernen Getreideverarbeitung und Getreidechemie . . . . . . . . . . . . . . . . 158

Rauhe Sitten bei Tisch . . . . . . . . . . . . . . 159

Rezepte - Anno dunnemals . . . . . . . . . . . . . . . . . . 159

50000 Erfassungsbelege für das Informationssystem ,,Nahrung und Ernährung des Menschen" . . . . . . . . . . . . . . . . 160

Tagungsankündigungen . . . . . . . . . . . . . . . . . . . . 160

Letzte Umschlagseiten: Kalorienreduzierte Werkessen

\section{Bezugsmöglichkeiten :}

In der DDR über den Postzeitungsvertrieb, übor Buchhandlungen oder den A kademie-Verlag

Im sozialistischen Ausland über den zuständigen Postzeitungsvertricb oder eine Buchhandlung für fremdsprachige Literatur

In der BRD und Westberlin uber eine Buchhandlung oder die Auslieferungsstelle KUNST UND WISSEN, Erich Bieber, 7 Stuttgart 1, Wilhelmstraße 4-6

In österreleh über den Globus-Buchvertrieb,

1201 Wien, Höchstädtplatz 3

Im übrigen Ausland über den Internationalen Buch- und Zeitschriftenhandel; über den Buchexport, Volkseigener Außenhandelsbetrieb der Deutschen Demokratischen Republik, DDR-701 Leipzig, Postfach 160, oder über den Akademie-Verlag.

DDR-107 Berlin, Leipziger Str. $3-4$

Hinwelse für Autoren

Dic Manuskripte sind in Original-Maschinenschrift einscitig und zweizeilig der Redaktion einzureichen. Pro Zeile 35 oder 70 Anschläge. Der Umfang der Manuskripte sollte mit der Redaktion vereinbart sein. Abbildungen, Tabellen und Zwischentitel sind erwünscht. Es ist zweckmäßig, die Arbeit mit einer kurzen Zusammenfassung oder mit Schlußfolgerungen sowie mit Empehlungen für die Praxis abzuschließen.

Dic Redaktion bchält sich eine Uberarbeitung der angenommenen Manuskripte vor; größere Änderungen nur mit Einverständnis des Autors. Die Beiträge werden honoriert, Sonderdrucke nicht hergestellt. 